\title{
Caracterização de Géis Termorreversíveis de SEBS
}

\author{
Antonio José F. Carvalho \\ Instituto de Química de São Carlos, Departamento de Físico-Química, USP
}

Resumo: Géis termorreversíveis preparados a partir de um copolímero em bloco de estireno - etileno/ butileno - estireno (SEBS) e polietileno de baixa densidade dissolvidos em óleos minerais parafínicos e óleos polibutênicos, bem como em suas misturas, foram preparados e caracterizados por calorimetria diferencial de varredura (DSC) e análise dinâmico mecânica (DMA). Os termogramas obtidos por DMA mostraram um comportamento bastante distinto em função do óleo utilizado como solvente. O valor de Tg e o módulo de estocagem, G' correspondente à temperatura de "onset" variaram de 25 a $47^{\circ} \mathrm{C}$ e $0,25 \mathrm{a} 9 \mathrm{MPa}$, respectivamente. Os géis compostos com óleos parafínicos apresentaram os maiores valores de Tg e de G'. As análises de DSC revelaram um pico endotérmico que foi designado como sendo de dissolução, entre $75^{\circ} \mathrm{C}$ e $90^{\circ} \mathrm{C}$ e o correspondente pico exotérmico $\left(60^{\circ} \mathrm{C}\right.$ e $\left.40^{\circ} \mathrm{C}\right)$ verificado durante o resfriamento. Esse processo de dissolução foi atribuído aos processos de fusão e solvatação dos cristalitos de polietileno. As transições observadas por DMA estão relacionadas aos domínios de poliestireno do copolímero em bloco, em conjunto com as transições do polietileno e são muito afetadas pelo tipo de solvente. Os géis estudados apresentam dois mecanismos distintos de gelificação, via formação de cristalitos de polietileno e via formação de agregados de poliestireno dos blocos terminais do SEBS. O efeito dos óleos aromáticos e naftênicos sobre o módulo dos materiais foi relacionado com um aumento na interação entre os agregados de poliestireno e a fase etileno-butileno.

Palavras-chave: Gel, termorreversível, copolimero em bloco, análise térmica, DSC, DMA, SEBS

\section{Characterization of Thermoreversible Gels of Sebs}

Abstract: Thermoreversible gels of styrene-ethylene/butylene-styrene block copolymers (SEBS) and low density polyethylene dissolved in mineral paraffinic and polybutene oils, as well its mixtures, were prepared and characterized by differential scanning calorimetry (DSC) and dynamic mechanical analysis (DMA). DMA analysis showed distinct behavior depending on the solvent used. The Tg value and storage modulus (G') at onset temperature ranges were 25 to $47^{\circ} \mathrm{C}$ and $0.25 \mathrm{MPa}$ to $9 \mathrm{MPa}$, respectively. The gels prepared with paraffinic oil showed the highest values of Tg and G'. DSC analysis showed an endothermic transition from $75^{\circ} \mathrm{C}$ to $90^{\circ} \mathrm{C}$ and its correspondent exotherm during cooling from $60^{\circ} \mathrm{C}$ to $40^{\circ} \mathrm{C}$. This transition was designated as dissolution due to fusion and solvation process of polyethylene crystallites. The transitions observed in DMA analysis depend on the solvent and are related to polystyrene domains of the block copolymer and to the polyethylene transitions and are affected by the oil used as solvent. The studied gels showed two different mechanism of gel formation, polyethylene crystallites and polystyrene aggregates. The effect of aromatic and naphthenic oils on the modulus was related to the improvement of interaction between the polystyrene domains and the ethylene/butylene phase.

Keywords: Gel, block copolymer, thermoreversible, thermal analysis, DSC, DMA, SEBS.

\section{Introdução}

Embora os géis sejam materiais muito conhecidos e façam parte do nosso cotidiano, só mais recentemente têm recebido maior atenção do ponto de vista acadêmico e industrial como uma nova classe de materiais com características bastante distintas dos materiais convencionais, que na sua maioria são sólidos. Em função da sua grande versatilidade e do incremento de sua aplicação onde os materiais convencio-

Antonio José F. Carvalho, Pirelli Cabos S.A., Av. Alexandre de Gusmão, 145, CEP: 09110-900, Santo André , SP. Endereço atual: USP, Instituto de Química, C.P. 780, CEP: 13560-970, São Carlos, SP. E-mail: felix@iqsc.sc.usp.br. 
nais apresentam grandes limitações, os géis têm substituído materiais sólidos e em alguns casos até líquidos e soluções. Atualmente os géis já encontram inúmeras aplicações, tais como purificação de água, membranas seletivas que podem operar como válvulas, cápsulas para liberação controlada de drogas, espessantes na indústria de alimentos, auxiliar na prospecção de petróleo, sistemas superabsorventes de água, sistemas de vedação dinâmica e estáticos e dispositivos eletro-mecânicos ${ }^{[1-3]}$ entre outras.

$\mathrm{Na}$ indústria de cabos de telecomunicação ${ }^{[3]}$, os géis encontram aplicação como bloqueadores ao ingresso de água ou outros agentes. Alguns desses géis têm a capacidade de absorver água aumentando de volume, e dessa forma preenchendo espaços vazios, selando o sistema. Outros são termorreversíveis, o que permite aplicá-los como um líquido viscoso que após resfriamento se apresentam como um gel que ocupa os espaços vazios preenchidos anteriormente pelo líquido, mantendo alguma mobilidade, porém estáveis a temperaturas relativamente altas. Neste caso, uma das principais características mecânicas do gel é a sua capacidade de se deformar plasticamente e permitir certa mobilidade, sem que ocorra o seu rompimento.

O termo "gel" tem sido muitas vezes utilizado de maneira imprópria para designar soluções concentradas de polímeros ou até materiais dessecados, como sílica gel. Além disso, termos relativos a géis têm sido introduzidos, tais como "weakgel", "quasigel", "temporary gel”, "pseudogel”, isogel", microgel" e "nanogel"[4], tornando ainda mais imprecisas as citações aos géis. Muitas dificuldades têm ocorrido também no que se refere às definições, onde podemos citar desde as mais clássicas como a de P.J. Flory ${ }^{[5]}$, até definições mais abrangentes como a de Ferry ${ }^{[6]}$. De acordo com a definição de Flory um gel é formado por uma rede polimérica unida por ligações covalentes e intumescida devido a presença de um solvente. Segundo a definição de Ferry ${ }^{[6]}$, um gel é um sistema substancialmente diluído, que não apresenta fluxo quando em repouso, ou seja no estado estacionário.

Uma proposta mais recente, postulada por Almdal ${ }^{[4]}$, é baseada principalmente em características fenomenológicas e no comportamento similar aos sólidos. Esta definição, ao contrário da definição de Ferry, estabelece uma escala de tempo para o estado em repouso. Segundo esta definição temos: a) Um gel consiste de dois ou mais componentes, um dos quais é um líquido, presente em quantidade substancial.

b)Um gel é um material com aspecto de sólido ou quase-sólido.

Para os géis com aparência de sólido, foi proposto por Almdal que as características dos mesmos devem ser definidas em termos de suas propriedades dinâmico-mecânicas, sendo que o módulo de estocagem G', deve apresentar um platô que se estenda a tempos da ordem de segundos e apresentar valor consideravelmente maior que o módulo de perda ${ }^{[4]}, \mathrm{G}$ ".

Para a maioria das aplicações industriais o tempo de relaxação deve ser suficientemente longo para não comprometer o sistema onde o gel foi aplicado. Além disso, em muitos casos, o gel deve suportar os esforços aos quais é submetido sem entrar no regime plástico ou deve entrar no regime plástico em tensões específicas, de modo a se acomodar mantendo o sistema íntegro. Para prever este comportamento as técnicas dinâmico-mecânicas são de extrema valia.

\section{Géis Poliméricos}

Géis poliméricos são constituídos de um solvente e pelo menos um polímero que estejam estruturado em forma de rede ${ }^{[7-10]}$. Os pontos de junção da rede podem ser permanentes formadas por ligações covalentes, sendo o gel neste caso, irreversível ou as junções podem ser de natureza física e o sistema é em geral reversível.

Os géis termorreversíveis apresentam pontos de junção como pontes de hidrogênio, zonas de cristalização e zonas de separação de fase líquido-líquido ${ }^{[8-10]}$. Estes géis sofrem mudanças drásticas de fase em função da temperatura, sendo que normalmente acima de uma determinada temperatura se comportam como soluções de polímeros. Soluções de polietileno em solventes como xileno, quando resfriadas formam géis termorreversíveis, onde os pontos de junção são cristalitos de polietileno ${ }^{[8]}$.

Géis reversíveis baseados em soluções resfriadas de polímeros sintéticos em óleos naturais e sintéticos são utilizados em aplicações industriais com o objetivo de propiciar vedação para impedir o ingresso de água ou outros agentes externos. As características de vedação e proteção para as quais estes géis são projetados dependem muito da estabilidade dos mes- 
mos em função do tempo. Esta estabilidade está diretamente associada à temperatura, especialmente no caso dos géis termorreversíveis. Um problema relativamente freqüente com os géis físicos é a sua instabilidade ao longo do tempo, que mais comumente se manifesta sob a forma de separação de solvente, podendo acarretar perda de função do mesmo. Esses problemas podem ser previstos através do relacionamento de características de estabilidade dos géis com suas características térmicas e mecânicas. Os ensaios dinâmicos mecânicos podem fornecer informações acerca da interação entre os componentes do sistema e da estabilidade do gel e, portanto, dar uma indicação do comportamento do gel em função do tempo e das condições de temperatura e tensão às quais é submetido.

Copolímeros em bloco do tipo MXM, onde M é um bloco de poli(metacrilato de metila) e X é polibutadieno ou polibutadieno hidrogenado e copolímeros do tipo SBS ou SEBS, tem sido estudados como agentes de formação de géis por geleificação física ${ }^{[11,12]}$. Os copolímeros em bloco do tipo SEBS (Kraton G - Shell Chemical) têm sido utilizados em inúmeras aplicações, inclusive em géis termorreversíveis ${ }^{[3]}$. Esses copolímeros apresentam blocos de poliestireno nas extremidades da cadeia separados por um bloco central de copolímero etileno-butileno, que será denominado fase borracha. O poliestireno dos blocos das extremidades não é compatível com a fase borracha e tenta se separar do bloco central, mas é impedido devido às ligações químicas com esta fase. Como resultado os blocos de poliestireno se movem para longe da fase borracha formando domínios de poliestireno através da fase borracha. Uma representação idealizada e simplificada deste arranjo é apresentada na Figura $1^{[11]}$.

As regiões discretas de poliestireno são representadas como esferas dentro de uma fase contínua formada pelo entrelaçamento das porções etileno/ butileno.

À temperatura ambiente, os domínios de poliestireno formados agem como ligações físicas e o sistema se comporta similarmente a uma borracha vulcanizada. Quando os domínios de poliestireno são amolecidos as moléculas não estão mais presas, o polímero pode ser processado como termoplástico ou dissolvido em um solvente apropriado para formar uma solução. No caso de géis formados por SEBS, os demais componentes, polietileno e o solvente (óleo) irão se entrelaçar à fase de etileno-butileno apresentando

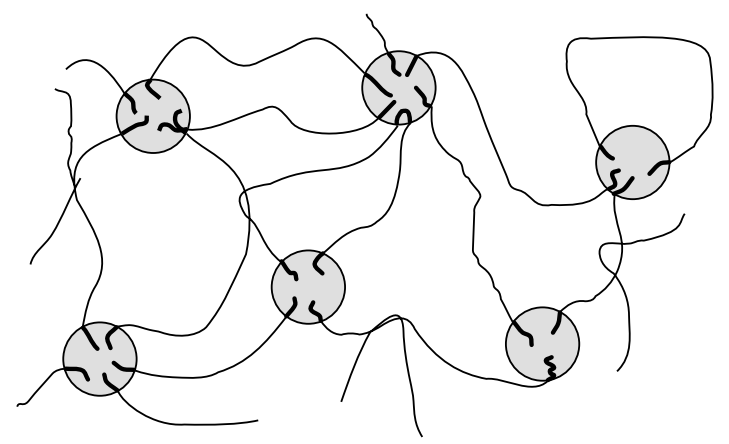

Figura 1. Representação simplificada da estrutura de um copolímero em bloco do tipo SEBS ${ }^{[11]}$.

interações dependentes da afinidade destes materiais e a fase etileno-butileno. Poderão também interagir com os domínios de poliestireno alterando as suas propriedades e consequentemente as propriedades do gel.

Neste trabalho, géis compostos de um copolímero em bloco estireno-etileno/butileno-estireno (SEBS) e polietileno de baixa densidade, em óleos minerais e poliisobutênicos foram caracterizados através de calorimetria diferencial de varredura, DSC, e análise dinâmico-mecânica, DMA. Os resultados de DMA foram interpretados tendo em vista a definição de gel proposta por Almdal ${ }^{[4]}$, levando-se em conta o efeito dos solventes utilizados sobre as propriedades mecânicas e térmicas.

\section{Experimental}

\section{Materiais}

Foi utilizado um copolímero em bloco estirenoetileno/butileno-estireno de nome comercial Kraton G, (Shell Chemical), polietileno de baixa densidade com índice de fluidez (MFI) de $14 \mathrm{~g} / \mathrm{min}$, óleos minerais parafínicos com $60 \%$ de parafínicos e com $98 \%$ de parafínicos, e poliisobutilenos líquidos (PIB) com massa molecular média em número de 330 e 500 daltons, (Polibutenos do Brasil S.A.). Em todas as misturas foi adicionado $0,2 \%$ de antioxidante "Pentaeritritol - tetrakis [3-(3,5-di -tercbutil-4hidroxifenil)-propionato]" para prevenir a oxidação durante o processo de preparação dos géis.

O tipo de óleo e suas misturas, utilizadas em cada composição são apresentados na Tabela 1.

\section{Preparação dos Géis.}

Os polímeros e o antioxidante foram dispersos no óleo ou mistura de óleos aquecidos e mantidos a $135^{\circ} \mathrm{C}$ 
Tabela 1. Tipo de óleo empregado na preparação dos géis de SEBS

\begin{tabular}{cc}
\hline Amostra & Óleo (Solvente) \\
\hline G1 & Óleo mineral $60 \%$ parafínico \\
G2 & Óleo mineral $98 \%$ parafínico \\
& Óleo mineral $60 \%$ parafínico/ PIB \\
G3 & Mn $330-1 / 2$ a $(\mathrm{m} / \mathrm{m})$ \\
& Óleo mineral. $60 \%$ parafínico $/ \mathrm{PIB}$ \\
G4 & Mn $330-1 / 8(\mathrm{~m} / \mathrm{m})$ \\
G5 & PIB Mn $330 / \mathrm{PIB} \mathrm{Mn} 500-8 / 1(\mathrm{~m} / \mathrm{m})$ \\
\hline
\end{tabular}

a Proporções em peso de cada óleo.

A composição dos géis, no que se refere às quantidades de cada polímero foi mantida inalterada.

até que se obtivesse uma solução. A solução foi derramada sobre uma placa de vidro e deixada esfriar para solidificar à temperatura ambiente. Foram obtidas placas do material com espessura de aproximadamente $2 \mathrm{~mm}$.

\section{Calorimetria Diferencial de Varredura, DSC}

As medidas de calorimetria diferencial de varredura, DSC, foram realizadas em um equipamento Perkin-Elmer DSC-7. As amostras foram analisadas em panela de alumínio fechadas. Como referência utilizou-se uma panela de alumínio vazia. As análises foram realizadas em atmosfera de nitrogênio, com fluxo de $30 \mathrm{ml} / \mathrm{min}$. O programa de temperatura utilizado foi: temperatura inicial $30^{\circ} \mathrm{C}$, tempo inicial 6 minutos, taxa de aquecimento $10^{\circ} \mathrm{C} / \mathrm{min}$, temperatura final $160^{\circ} \mathrm{C}$ e tempo final 3 minutos. $\mathrm{O}$ resfriamento foi realizado a $10^{\circ} \mathrm{C} / \mathrm{min}$, até $30^{\circ} \mathrm{C}$.

Este ciclo foi repetido três vezes. O primeiro ciclo teve como objetivo a padronização da história térmica das amostras e uniformizar o contato das mesmas com a panela. O segundo ciclo foi utilizado nos cálculos das temperaturas de transição e energias envolvidas. O ensaio foi repetido toda vez que o segundo e terceiro ciclo apresentaram divergências entre si.

\section{Análise Dinâmico-Mecânica, DMA}

As análises foram realizadas em um equipamento Polymer, no modo de cisalhamento, a uma freqüência de $1 \mathrm{~Hz}$ e uma taxa de aquecimento de $10^{\circ} \mathrm{C} / \mathrm{min}$, entre $-60^{\circ} \mathrm{C}$ e $100^{\circ} \mathrm{C}$.

\section{Resultados e Discussão}

\section{Calorimetria Diferencial de Varredura}

O resultado da análise de DSC de G1 é apresentado na Figura 2. Este termograma apresenta três ciclos de aquecimento e resfriamento. Em todos os casos os picos de dissolução e solidificação são bem definidos. Os termogramas dos demais materiais se apresentaram muito similares ao de G1. Os dados de temperatura e energias correspondentes envolvidas no processo de dissolução e solidificação são apresentados na Tabela 2.

Géis de poliacrilonitrila apresentam termogramas de DSC com a presença de pico endotérmico de fusão que foram atribuídos à dois processos $\operatorname{distintos}^{[8]}$ : quebra das ligações na rede cristalina, que é acompanhado de um calor de fusão, $\Delta \mathrm{H}$ fusão, e solvatação dos constituintes do cristal fundido, que é acompanhado por calor parcial de solvatação, $\Delta \mathrm{H}$ solvatação. As quantidades termodinâmicas são funções do estado, e foram escritas como calor parcial de dissolução ${ }^{[8]}$ :

$$
\Delta \mathrm{H} \text { dissolução }=\Delta \mathrm{H} \text { fusão }+\Delta \mathrm{H} \text { solvatação }
$$

Portanto as variações de entalpia verificadas por DSC correspondem ao calor de dissolução e não ao

Tabela 2. Valores determinados de $\Delta H$ de dissolução, $\Delta H$ de solidificação e respectivas temperaturas de pico

\begin{tabular}{ccccc}
\hline Amostras & $\begin{array}{c}\text { T pico "dissolução" } \\
\left({ }^{\circ} \mathbf{C}\right)\end{array}$ & $\begin{array}{c}\Delta \mathbf{H} \text { "dissolução" } \\
\text { J/g de gel }\end{array}$ & $\begin{array}{c}\text { T pico "solidificação" } \\
\left({ }^{\circ} \mathbf{C}\right)\end{array}$ & $\begin{array}{c}\Delta \mathbf{H} \\
\text { "solidificação" } \\
\text { J/g de gel }\end{array}$ \\
\hline G1 & 76,9 & 8,5 & 53,5 & $-8,4$ \\
G2 & 77,5 & 6,8 & 52 & $-6,5$ \\
G3 & 77,5 & 6,5 & 53 & $-5,8$ \\
G4 & 78 & 7,5 & 54,2 & $-7,2$ \\
G5 & 79,7 & 8,3 & 53,3 & $-7,8$ \\
\hline
\end{tabular}

Valor médio para $\Delta \mathrm{H}$ de dissolução é $7,5 \mathrm{~J} / \mathrm{g} \pm 0,9 \mathrm{~J} / \mathrm{g}$. 


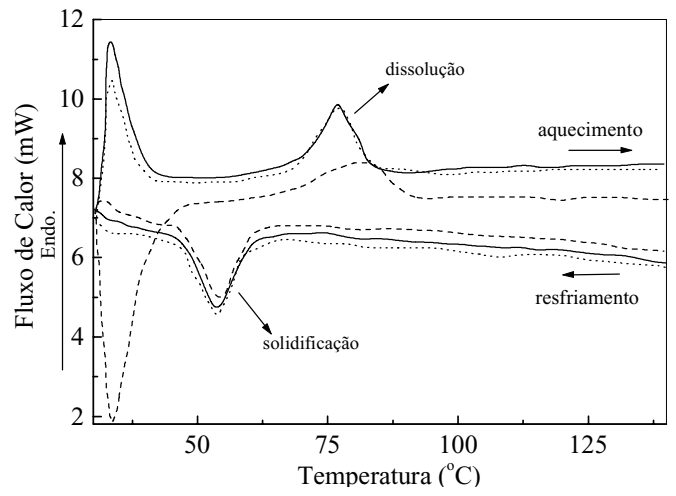

Figura 2. Curvas obtidas por análise de calorimetria diferencial de varredura para o gel G1.

calor de fusão. Se considerarmos que o tipo de solvente não interfere na formação do cristal, as diferenças nos valores do calor de dissolução observadas, se devem às variações no calor de solvatação devido ao tipo de solvente em questão. Os calores de dissolução observados apresentaram valor médio da ordem de 7,5 J/g de gel, o que corresponde a $125 \mathrm{~J} / \mathrm{g}$ de polietileno, considerando a concentração do polietileno no gel. O calor de fusão determinado para o polietileno utilizado foi de $65 \mathrm{~J} / \mathrm{g}$, portanto bastante inferior ao valor determinado para o polietileno no gel. Essa diferença deve-se ao calor de solvatação ou a um possível efeito do solvente sobre a formação dos cristalitos de polietileno. Para determinarmos esses valores com precisão seria necessário obter o calor de solvatação de uma amostra de polietileno totalmente amorfo, nos solventes estudados.

Os géis $\mathrm{G} 1$ e $\mathrm{G} 5$ apresentam valores de $\Delta \mathrm{H}$ de dissolução maiores que os demais géis. G1 contém como solvente um óleo parafínico com $40 \%$ de naftênicos e aromáticos, o que o diferencia de G2 e G5, que é composto por um óleo de mesma natureza química que os óleos dos géis G3 e G4, porém de maior massa molecular e, portanto de maior viscosidade. Tanto a natureza química como a massa molecular pode afetar o $\Delta \mathrm{H}$ de dissolução.

A importância desses processos reside no fato de que o processo de cristalização do polietileno contribui para o processo de geleificação, devido à formação de cristalitos de polietileno, que agem como ligações cruzadas, de caráter físico e, portanto reversíveis.

\section{Análise Dinâmico-Mecânica}

Os resultados dos ensaios de DMA são apresentados na Tabela 3. Nas Figuras 3, 4 e 5 são apresentados os registros de DMA para a tan $\delta$, módulo elástico, G', e módulo de perdas, G', respectivamente, dos géis estudados.

As amostras G1 e G2, que são compostas por óleo parafínico, apresentam um pico de $\tan \delta$ pronunciado a $41,4^{\circ} \mathrm{C} \mathrm{e} 46,4^{\circ} \mathrm{C}$, respectivamente (ver Figura 3). Essas transições correspondem à transição vítrea $\mathrm{Tg}(\mathrm{Tg}$, definida como a temperatura no máximo da $\tan \delta$ correspondente a transição $\alpha$ ), dos agregados de poliestireno, deslocadas, para valores mais baixos devido ao efeito plastificante do óleo.

A amostra G1 apresentou pico de $\tan \delta$, com intensidade muito superior aos demais materiais, que apresentaram valores de $\tan \delta$ com magnitude cerca de 10 vezes inferior. $O$ valor da temperatura de transição vítrea de G2 é maior que o valor de G1, mostrando que o óleo totalmente parafínico apresenta um menor efeito plastificante para os agregados de poliestireno. Os géis G1 e G2 apresentaram um pico de amortecimento com um ombro de um possível pico de dispersões secundárias.

Os picos de $\tan \delta$ dos géis G3, G4 e G5 são muito

Tabela 3. Valores de Temperatura de "onset", Tg, módulo na temperatura de onset e Tan $\delta$

\begin{tabular}{|c|c|c|c|c|c|c|}
\hline \multirow{2}{*}{ Amostra } & \multirow{2}{*}{$\begin{array}{c}\text { T"onset" } \\
\left({ }^{\circ} \mathrm{C}\right)\end{array}$} & \multicolumn{2}{|c|}{$\begin{array}{c}\text { Temperatura dos picos de } \\
\text { Tan } \delta\left({ }^{\circ} \mathrm{C}\right)\end{array}$} & \multirow{2}{*}{$\begin{array}{c}\text { Módulo na } \\
\text { temperatura de } \\
\text { "onset" } \\
\text { (MPa) }\end{array}$} & \multicolumn{2}{|c|}{ Magnitude de $\operatorname{Tan} \delta$} \\
\hline & & (Tg) $\alpha$ & $\beta *$ & & $\alpha$ & $\beta$ \\
\hline G1 & -20 & 41,4 & 30 & 9 & 2,30 & 1,30 \\
\hline G2 & -10 & 46,4 & 25 & 0,7 & 0,56 & 0,40 \\
\hline G3 & $<-40$ & 44 & 10 & 0,25 & 0,17 & 0,17 \\
\hline G4 & $<-40$ & 20 & - & 0,8 & 0,22 & 0,35 \\
\hline G5 & $<-40$ & 25 & 2,5 & 0,3 & 0,25 & 0,25 \\
\hline
\end{tabular}

* As transições designadas por $\beta$, com exceção de G1 se apresentam de modo muito discreto ou sob a forma de um ombro. 


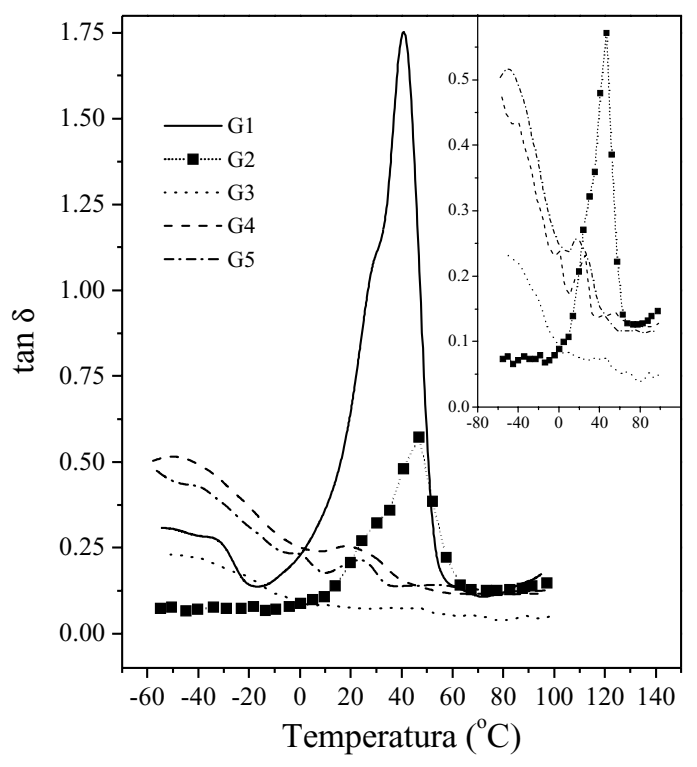

Figura 3. Curvas obtidas por análise dinâmico-mecânica para tan $\delta$, dos géis G1, G2, G3, G4 e G5, incluindo a expansão das curvas em separado.

discretos e na forma de ombros. Com o abaixamento da temperatura o valor de tan $\delta$ aumenta até um máximo. Uma possível interpretação para esse fato é a ocorrência de separação de fase no sistema, gerando uma fase rígida e uma líquida. A fase líquida amortece as respostas dos agregados de poliestireno, tornando difícil a detecção de suas transições. Na prática se observa, após algumas semanas, a separação de óleo do gel. Este fenômeno ocorre em função de uma pior interação entre as fases

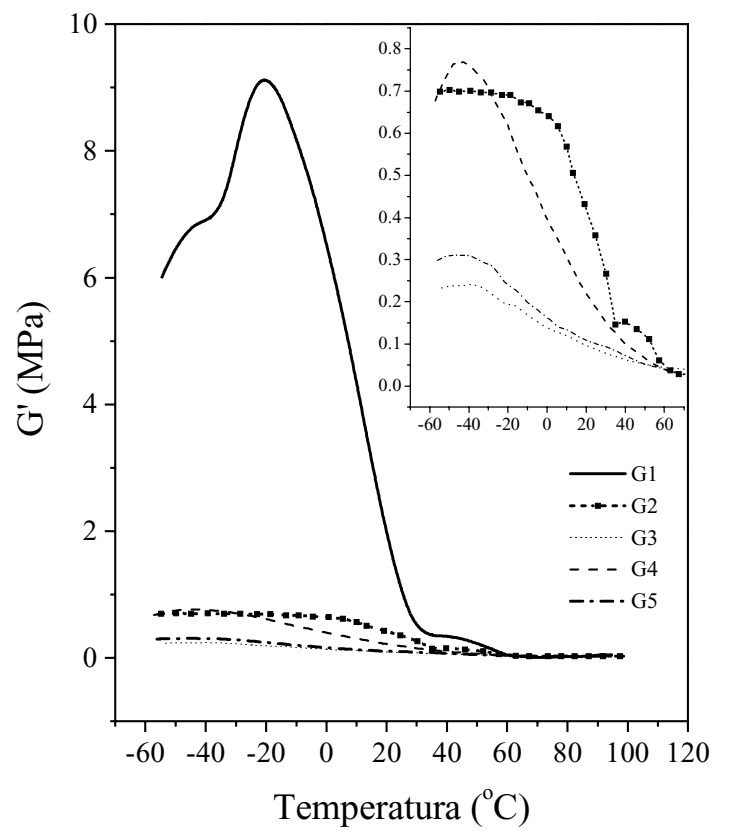

Figura 4. Curvas obtidas por análise dinâmico-mecânica para o módulo elástico, G', dos géis G1, G2, G3, G4 e G5, incluindo a expansão das curvas em separado. olefínicas, isto é, as porções etileno/butileno do SEBS e os óleos polibutênicos. Do ponto de vista fenomenológico os géis compostos com óleos polibutênicos não podem também ser considerados géis, considerando a definição proposta por Almdal ${ }^{[4]}$, pois não apresentam módulo de estocagem (elástico) com platô a temperatura ambiente com magnitude visivelmente superior ao módulo de perdas.

O óleo e o polietileno, em função de suas baixas polaridades estão dispersos na fase borracha, onde estão entrelaçados com as cadeias de etileno-butileno. $\mathrm{O}$ módulo elástico desta fase será, portanto muito afetado em função do tipo de interação entre o óleo e a fase borracha. Quanto melhor o solvente, do ponto de vista termodinâmico, maior será o modulo elástico. Isto explicaria em parte a grande diferença de módulo verificada entre os géis com óleo parafínico e poliisobutilênicos. Contudo, não explicaria a diferença de módulo entre G1 e G2, ambos com óleo parafínico. O gel G2 é composto por um óleo totalmente parafínico e G1 é composto por um óleo parcialmente parafínico, que contêm substancial quantidade de óleos aromáticos e naftênicos. O óleo com maior teor de naftênicos e aromáticos, embora seja um pior solvente para a fase borracha, irá estabelecer maior interação com os domínios de poliestireno, o que propiciaria um maior entrelaçamento entre as fases.

As temperaturas de "onset" para os géis à base de óleo polibutênico são bastante inferiores àquelas dos géis

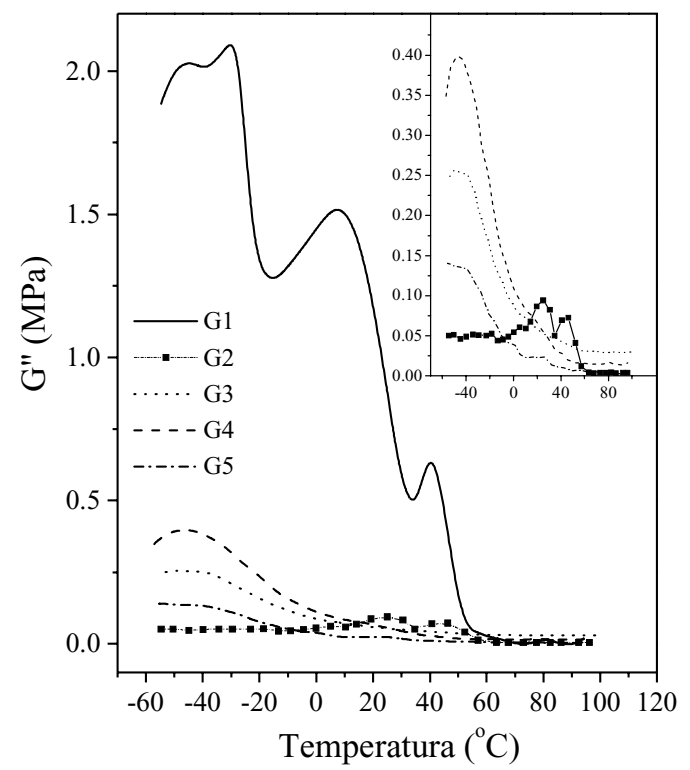

Figura 5. Curvas obtidas por análise dinâmico-mecânica para o módulo de perdas, G", dos géis G1, G2, G3, G4 e G5, incluindo a expansão das curvas em separado. 
compostos por óleo parafínico. Mesmo no caso de G3 e G4, composto por uma mistura dos óleos parafínico e polibutênico, prevalece o efeito do óleo polibutênico sobre as propriedades dinâmico-mecânicas. Nestes casos não existe um patamar de G' bem definido e superior a G", que caracterize um sólido. Estes "géis" apesar de aparentemente se assemelharem a G1 e G2 não são estáveis e tendem a se desagregar com separação de óleo, se assemelhando a uma esponja encharcada que sob o efeito do próprio peso perde líquido.

Os resultados da análise de DMA podem ser relacionados com as aplicações práticas destes materiais, visto que os mesmos devem ser dimensionalmente estáveis durante o tempo, numa faixa de temperatura que engloba a temperatura ambiente. Além disso, efeitos de separação de óleo que normalmente são percebidos apenas após semanas podem ser previstos pela análise dos dados de DMA.

Embora os materiais estudados apresentem características físicas muito similares entre si, eles apresentam profundas diferenças de comportamento dinâmico mecânico. A técnica de DSC permite apenas uma melhor compreensão dos efeitos de temperatura sobre os cristalitos de polietileno, que no caso específico dos materiais estudados não são preponderantes sobre as propriedades físicas dos "géis" que são basicamente governadas pelo copolímero em bloco de SEBS. Embora o tipo e composição dos solventes estudados tenham tido um pronunciado efeito sobre a fusão e cristalização dos cristalitos, o mecanismo de formação de gel que determina as características dinâmico-mecânicas do material são os agregados de poliestireno do SEBS.

Géis muito similares quanto ao aspecto visual quando analisados por técnicas de análise térmica revelaram profundas diferenças que podem ser correlacionadas com o comportamento destes em função do tempo, o que pode ajudar muito na escolha de materiais e composições para aplicações específicas.

\section{Conclusões}

A presença de porções aromáticas e naftênicas no óleo parafínico é responsável pela grande diferença de módulo entre os géis G1 e G2, devido a uma maior interação entre a fase borracha e os agregados de poliestireno. Os materiais compostos com óleos polibutênicos, G3, G4 e G5, apresentaram três picos de $\tan \delta$, de baixa magnitude, e valores baixos de módulo de estocagem, devido a pouca afinidade dos óleos polibutênicos e a fase etileno/ butileno do SEBS.

As técnicas de DSC e DMA em conjunto podem ser de grande valia no estudo de géis poliméricos, podendo prever seu comportamento em função do tempo, a partir de dados gerados com rapidez, a partir de pequenas quantidades de amostras. Somente o gel G1 apresentou módulo elástico G' superior a G” em uma faixa relativamente larga de temperatura, o que o caracteriza como um gel, segundo definição baseadas em características físicas.

\section{Referências Bibliográficas}

1. Djabourov, M. - Polymer International, p. 25, 135 (1991).

2. Osada, Y. \& Ross-Murphy, S. B. - Scientific American, may, p. 42 (1993).

3. Mitchell, D.; Sabia, R. - "Development, Characterization, and Performance of an Improved Cable Filing Compound", in: International Wire \& Cable Symposium Proceedings, p. 15, (1980).

4. Almdal, K.; Hvidt, J. D. S.; Kramer, O. - Polymer Gels and Networks, 1, p. 5 (1993).

5. Flory, P. J. - " Principles of Polymer Chemistry” Cornell University Press, Ithaca, NY, (1978).

6. Ferry, J. D. - “Viscoelastic Properties of Polymers", John Wiley \& Sons, New York, (1980).

7. Tager, A. - "Physical Chemistry of Polymers", Mir Publishers, Moscow, (1978).

8. Bashir, Z. - J. Appl. Polym. Sci., Part B: Polym. Phys., 30, p. 1299 (1992).)

9. Russo, P.S. - "A Perspective on Reversible gels and Related Systems - Reversible Polymeric Gels and Related Systems", in: ACS Symposium Series 350, American Chemical Society, Washigton DC, p1 (1987).

10. Hikmet, R.M.; Callister, S. ; Keller, A. - Polymer, 29, p. 1378 (1988).

11. Yu, J.M. \& Jérôme, R., Macromolecular Chemistry and Physics, 11, p. 3719 (1997).

12 Yu, J.M.; Blacher, S.; Brouers, F.;L’Homme, G. \& Jérôme, R., Macromolecules, 30, p. 4619 (1997).

Recebido: $30 / 07 / 98$

Aprovado: 22/11/99 